\title{
Nonparametric inference of stochastic differential equations based on the relative entropy rate
}

\author{
Min Dai ${ }^{1}$, Jinqiao Duan ${ }^{2}$, jianyu $\mathrm{Hu}^{3}$, and Xiangjun Wang ${ }^{4}$ \\ ${ }^{1}$ Wuhan University of Technology \\ ${ }^{2}$ Illinois Institute of Technology \\ ${ }^{3}$ Huazhong University of Science and Technology \\ ${ }^{4}$ Huazhong University of Science and Technology - Wuchang Campus
}

November 19, 2021

\begin{abstract}
The information detection of complex systems from data is currently undergoing a revolution,driven by the emergence of big data and machine learning methodology. Discovering governingequations and quantifying dynamical properties of complex systems are among central challenges. Inthis work, we devise a nonparametric approach to learn the relative entropy rate from observationsof stochastic differential equations with different drift functions. The estimator corresponding tothe relative entropy rate then is presented via the Gaussian process kernel theory. Meanwhile, thisapproach enables to extract the governing equations. We illustrate our approach in several examples.Numerical experiments show the proposed approach performs well for rational drift functions, notonly polynomial drift functions.
\end{abstract}

\section{Hosted file}

Nonparametric inference of stochastic differential equationsbased on the relative entropy rate.pdf available at https://authorea.com/users/447034/articles/546129-nonparametric-inference-ofstochastic-differential-equations-based-on-the-relative-entropy-rate

\section{Hosted file}

Nonparametric inference of stochastic differential equationsbased on the relative entropy rate.tex available at https://authorea.com/users/447034/articles/546129-nonparametric-inference-ofstochastic-differential-equations-based-on-the-relative-entropy-rate 

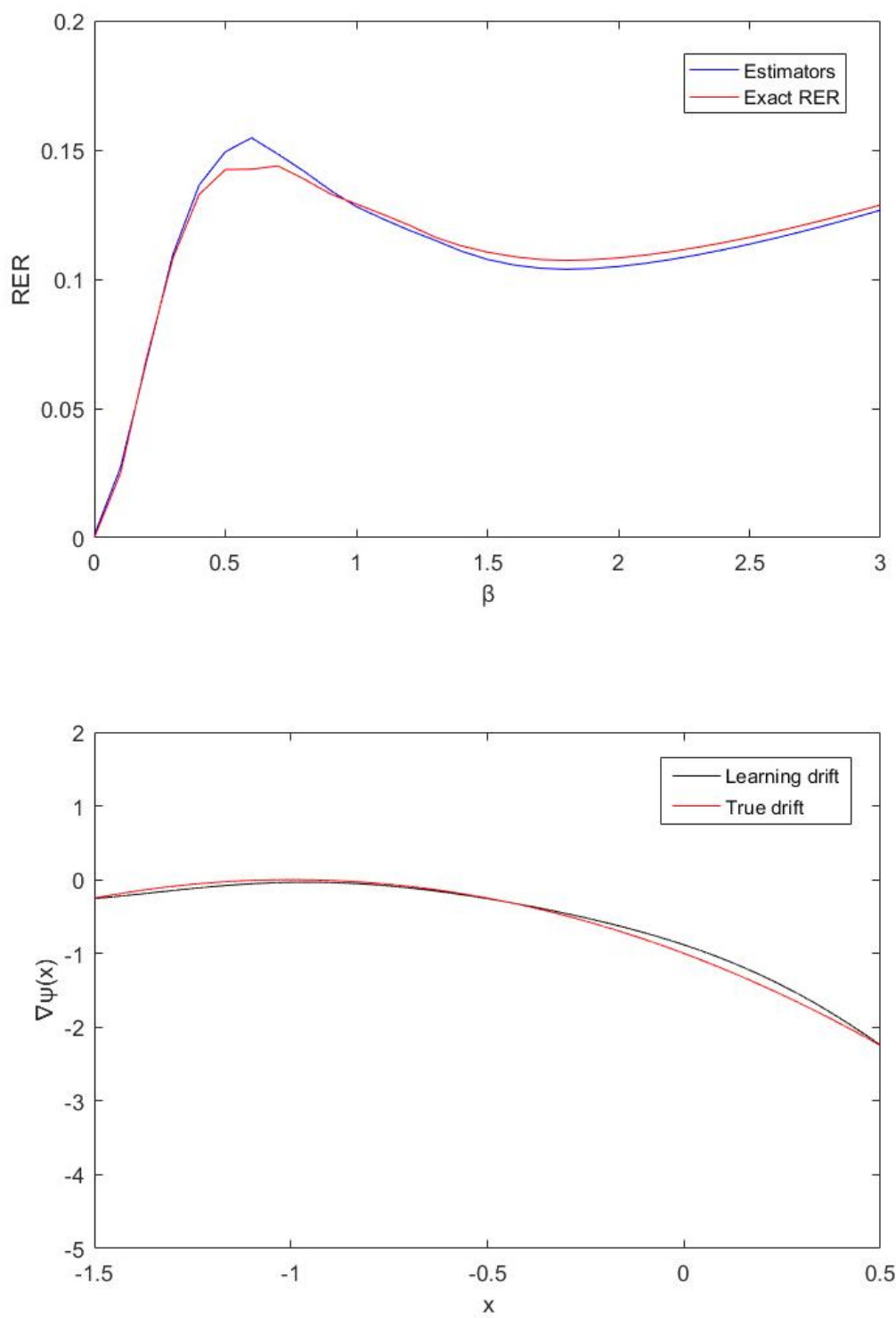

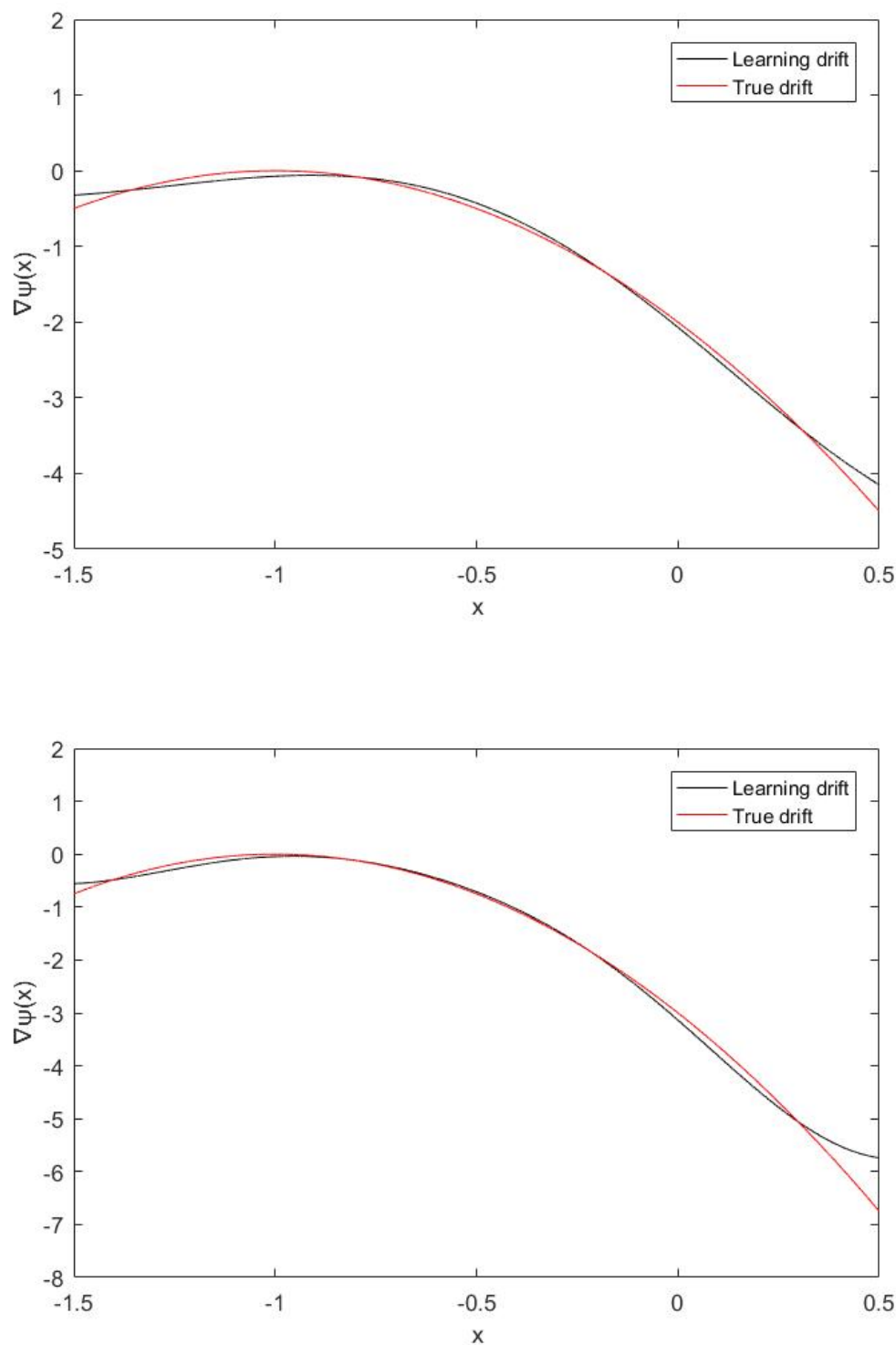

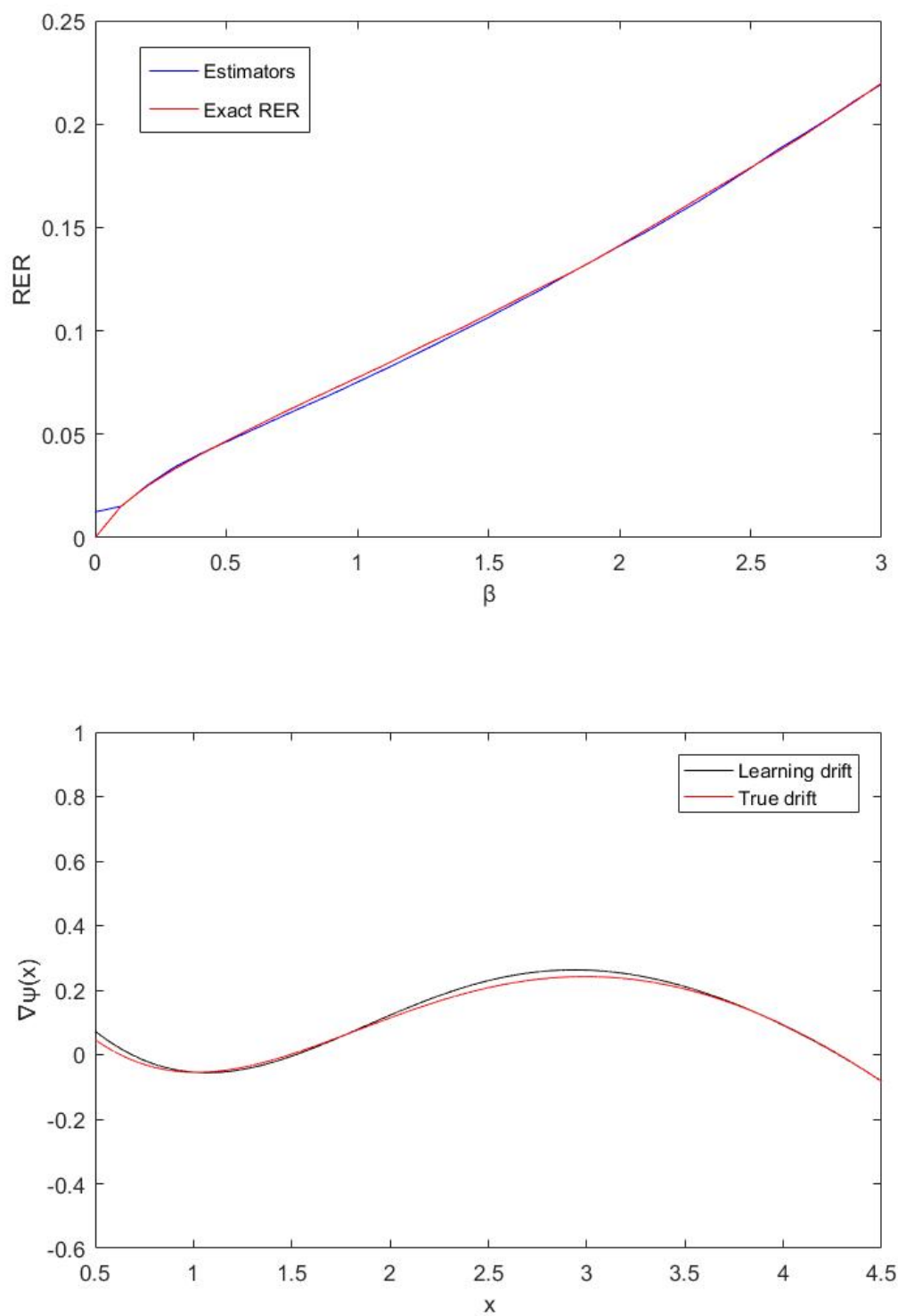

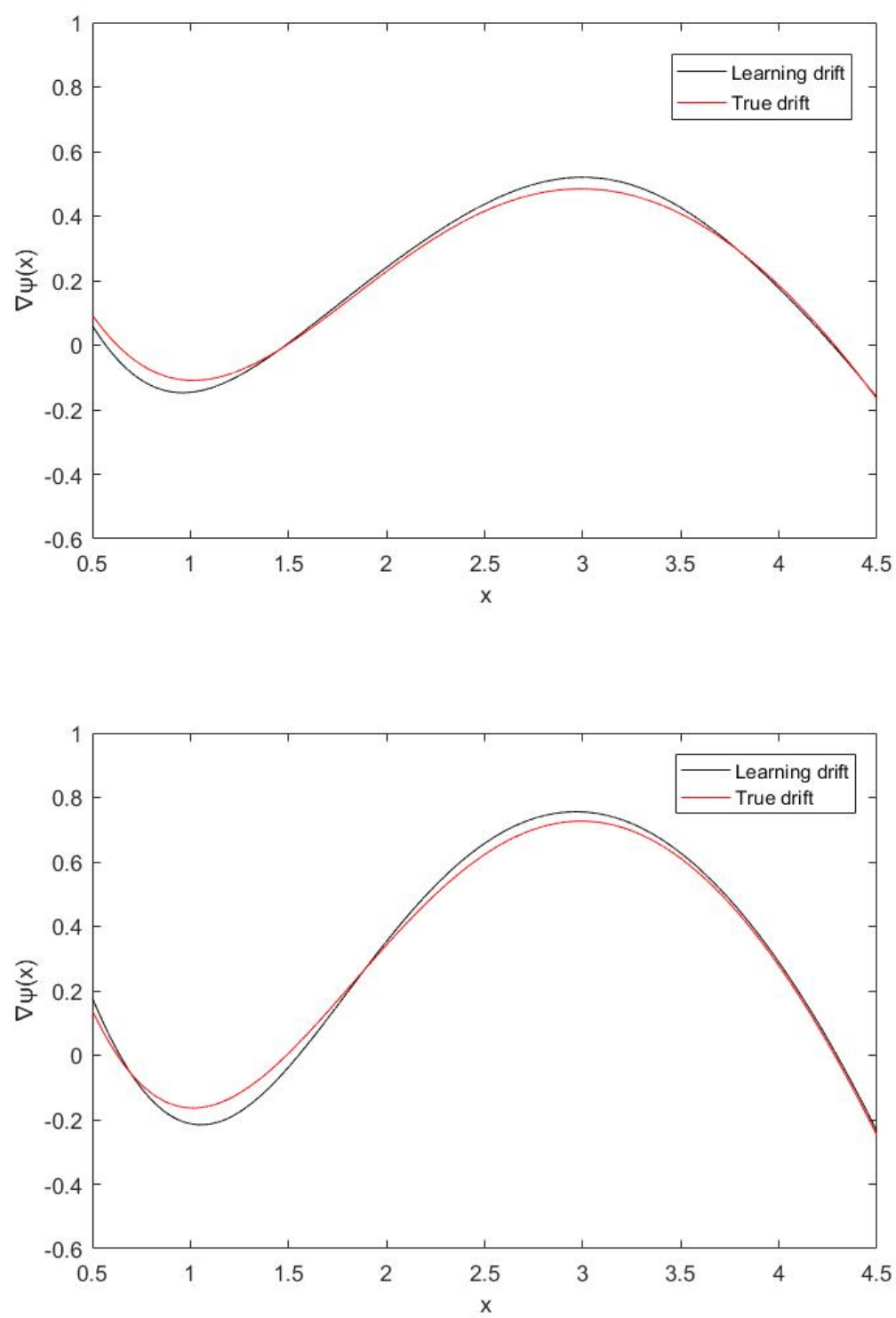

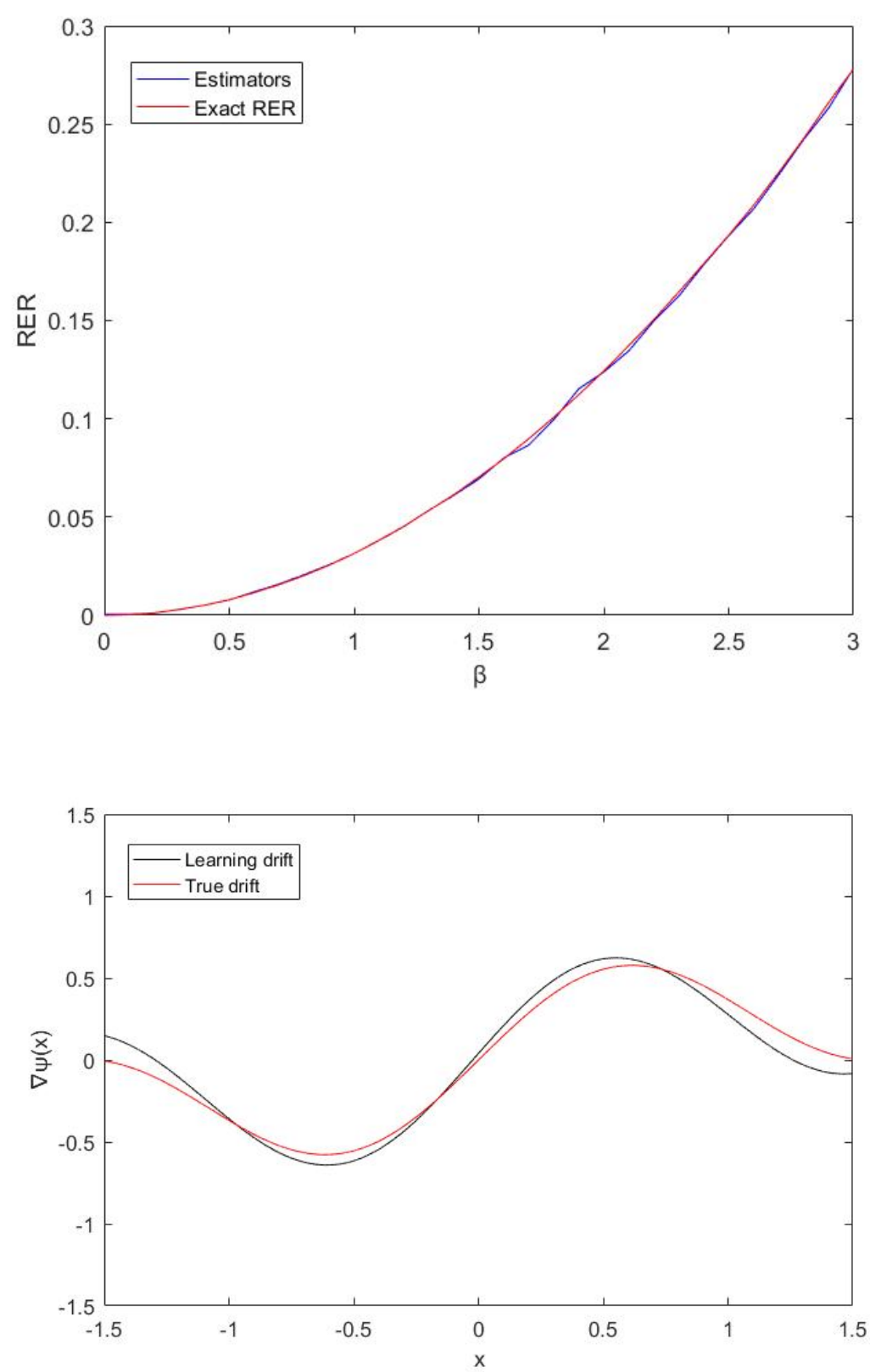

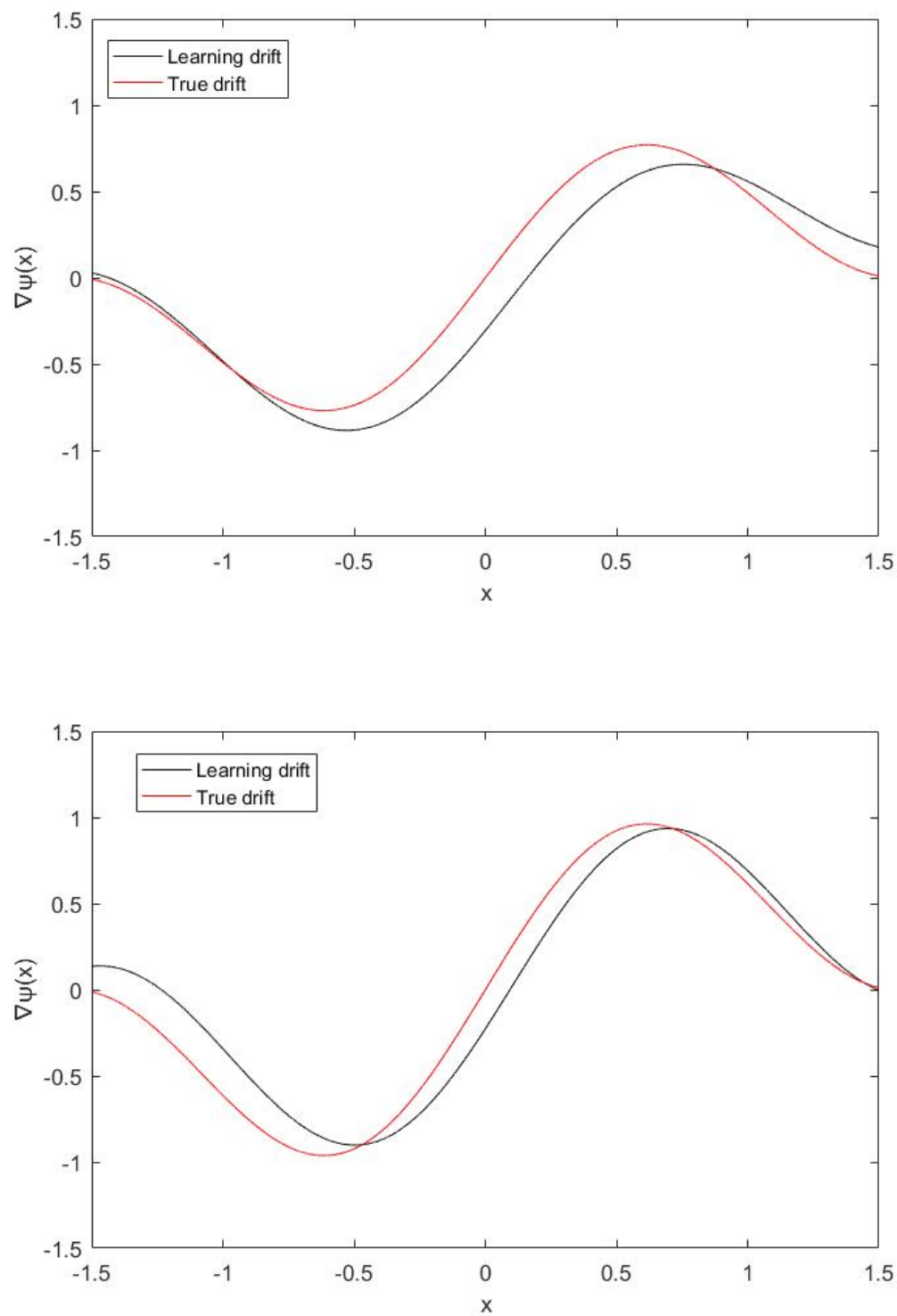